\title{
Cobertura de puericultura e fatores associados em São Luís (Maranhão), Brasil
}

\author{
Antônio Augusto Moura da Silva, Uilho Antônio Gomes, ${ }^{2}$ \\ Sueli Rosina Tonial ${ }^{1}$ e Raimundo Antonio da Silva ${ }^{1}$
}

RESUMO A assistência de puericultura é fundamental para a prevenção de diversas doenças durante os primeiros anos de vida da criança. O início precoce e a realização de pelo menos nove consultas no primeiro ano são metas desejáveis na assistência à criança. Inquéritos periódicos são fundamentais para que se possa acompanhar a obtenção destas metas. Em 1994, um estudo transversal utilizou amostragem por conglomerados em três estágios para avaliar cobertura de puericultura e fatores associados entre crianças em São Luís, Estado do Maranhão, Brasil. Para a coleta de dados, um questionário padronizado foi respondido pela mãe ou responsável pela criança. O percentual de recusas e ausências foi de $7 \%$. O presente trabalho analisa os dados relativos a 290 crianças entre 1 e 23 meses. As estimativas pontuais e por intervalo de confiança de $95 \%$ das proporções levaram em conta o efeito de desenho. Na estimativa da razão ajustada de prevalências empregou-se a regressão de Cox modificada para estudos transversais. A cobertura de puericultura para a população estudada foi de $80 \% ; 44 \%$ das crianças realizaram até seis consultas no primeiro ano de vida - o que está bem abaixo do recomendável com média de 7,8 consultas no primeiro ano. Oitenta e um por cento das crianças utilizaram o Sistema Único de Saúde para o atendimento, sendo de apenas $15 \%$ a utilização do segurosaúde. Após ajuste para fatores de confusão, os fatores "maior número de irmãos no domicílio" $e$ "pai com 4 ou menos anos de estudo" estiveram associados à não realização de consultas de puericultura. O fator "renda familiar" não permaneceu associado após o ajuste. O reforço às atividades educativas e, talvez, aos programas de planejamento familiar, é uma estratégia importante no sentido de aumentar a cobertura de puericultura no município.

A assistência em puericultura é fundamental para a prevenção de diversas doenças durante os primeiros anos de vida da criança. $O$ início precoce das consultas, de preferência no primeiro mês de vida, e a realização de

\footnotetext{
1 Universidade Federal do Maranhão, Departamento de Saúde Pública. Correspondência e pedidos de separatas devem ser enviados a Antônio Augusto Moura da Silva no seguinte endereço: Departamento de Saúde Pública, Universidade Federal do Maranhão, Rua Barão de Itapary 155 CEP 65020-070, São Luís, Maranhão, Brasil. E-mail: aasilva@elo.com.br

2 Universidade de São Paulo, Faculdade de Medicina de Ribeirão Preto, Ribeirão Preto, SP, Brasil.
}

pelo menos nove consultas no primeiro ano de vida são metas desejáveis na assistência à criança (1). Inquéritos periódicos são fundamentais para que se possa acompanhar a obtenção destas metas (2). $\mathrm{O}$ acesso à puericultura pode, inclusive, reduzir a necessidade de hospitalizações $(3,4)$.

No mundo, alguns inquéritos foram realizados com a finalidade de conhecer-se a cobertura de atendimento de crianças sadias. Em Santiago do Chile, em 1977, constatou-se uma média de 6,8 consultas de puericultura realizadas no primeiro ano de vida e de 2,1 controles no pré-escolar. As crianças de mães situadas nos dois extremos de renda e escolaridade realizaram maior número de consultas (5). Outra pesquisa realizada em Santiago do Chile, em 1983, demonstrou que as mães mais jovens (com menos de 25 anos) e com menor número de filhos tendiam a usar mais vezes os serviços de puericultura (6).

Em 1982, no Panamá, uma pesquisa nacional com crianças menores de 6 anos mostrou que $89 \%$ recebiam assistência em puericultura, sendo a maioria destas consultas realizada nos serviços públicos de saúde. O maior uso deste serviço foi verificado entre as crianças 
cujas mães tinham entre 25 e 34 anos, entre as crianças de famílias com menos filhos, crianças cujas mães tinham maior escolaridade e crianças cujas famílias tinham renda mais alta (7).

Em estudo realizado em Burkina Fasso, na África, os seguintes fatores estiveram associados à maior freqüência à puericultura: o tipo de serviço disponível na localidade (hospital, posto de saúde ou nenhum), maior escolaridade materna, menor distância da casa ao serviço de saúde, menor idade da criança e maior escolaridade paterna (8).

Nos Estados Unidos, crianças das classes desfavorecidas realizam menos consultas preventivas (3). Crianças sem qualquer forma de seguro-saúde representam, naquele país, 8 milhões. Estas crianças possuem um risco 1,5 vezes maior de não receber assistência em puericultura quando comparadas às demais. A posse de seguro-saúde é considerada um importante preditor da utilização dos serviços de saúde nos Estados Unidos (9).

São poucos os inquéritos populacionais realizados no Brasil sobre a cobertura e a qualidade da assistência de puericultura. Monteiro et al. (10) estudaram a cobertura e a qualidade da assistência em puericultura no Município de São Paulo, em amostra de crianças menores de 5 anos, em 1983 e 1984. A cobertura de puericultura para crianças de 12 a 59 meses foi quase universal, de 98\%; 65\% das crianças realizaram a primeira consulta preventiva nos dois primeiros meses de vida. Apesar da alta cobertura alcançada, levando-se em conta a realização de pelo menos uma consulta, a média de consultas no primeiro ano de vida foi de 7,7, abaixo da meta preconizada de nove. A desigualdade na época de início da puericultura foi grande: $84 \%$ das crianças de famílias cujos chefes tinham maior escolaridade (12 anos ou mais) iniciaram a puericultura precocemente, ao passo que apenas $46 \%$ das crianças de famílias com chefes que não haviam freqüentado a escola o fizeram (10).

Em Pelotas, Estado do Rio Grande do Sul, um estudo longitudinal com 6000 crianças, nascidas em 1982, veri- ficou que o número médio de consultas preventivas realizadas pelas crianças participantes nos últimos 12 meses antes da pesquisa era de 8,9. Esta média foi mais elevada para os grupos de alta renda (média de 10,4) do que para os de baixa renda (média de 7,5 ). Em 1993, a média caiu para 7,3 consultas e o diferencial segundo a renda familiar se manteve (11).

Campos (12), estudando uma amostra das crianças menores de 5 anos do município de Londrina, Estado do Paraná, Brasil, evidenciou cobertura praticamente universal da puericultura no município, onde $99 \%$ das crianças iniciaram a puericultura no primeiro mês de vida. A média de consultas no primeiro ano de vida foi de 10,8 , sendo de 7,7 para as crianças de famílias cujos chefes não freqüentaram escola e de 13,1 para as crianças de famílias com chefes de maior escolaridade (12).

O objetivo do presente trabalho é estimar a cobertura da assistência em puericultura entre crianças de 1 a 23 meses no Município de São Luís, Estado do Maranhão, Brasil, e analisar alguns fatores associados à não realização de consultas de puericultura, visando a contribuir para a identificação de barreiras ao uso destes serviços e para a implementação de medidas corretivas.

\section{MATERIAIS E MÉTODOS}

O Município de São Luís localiza-se na ilha do Maranhão, ao norte do Estado do Maranhão, tendo uma área de $518 \mathrm{~km}^{2}$. Em 1996, sua população era de 781068 habitantes. São Luís teve forte crescimento demográfico ao longo das últimas décadas, apesar da taxa de crescimento populacional estar diminuindo. A estrutura dos serviços de saúde, especialmente a rede de ambulatórios públicos, cresceu nos últimos anos (13).

Realizamos um estudo transversal, utilizando o método de amostragem por conglomerados em três estágios. No cálculo do tamanho amostral utilizou-se a fórmula $n=\left[\left(z^{2} \times p \times q\right) /\right.$ $\left.d^{2}\right] \times 2$ (correção para efeito de desenho) (14), onde $z^{2}$ reflete o nível de confiança adotado; $p$ é a proporção esperada de crianças que realizam consultas de puericultura, equivalendo à cobertura; $q$ equivale a $1-p$; e $d^{2}$ é a precisão absoluta desejada em torno da estimativa. Fixando-se o nível de confiança em $95 \%$ e a precisão absoluta desejada em torno da estimativa de 5\% (e como se estimaram muitas variáveis na mesma amostra), calculou-se o produto máximo de $p \times q(50 \%)$, no sentido de obter-se amostra de maior abrangência possível. $\mathrm{O}$ tamanho amostral necessário foi de 800 crianças. Esta amostra tem poder de $80 \%$ para detectar diferenças entre subgrupos acima de $12 \%$ para percentuais situados ao redor de $50 \%$, e acima de $6 \%$ para percentuais situados ao redor de 10 ou $90 \%$. Não foram utilizadas estimativas ponderadas pois, apesar de a amostragem não ter sido equiprobabilística, a diferença entre as estimativas ponderadas e não ponderadas foi, em todos os casos, inferior a $1 \%$.

No primeiro estágio foram sorteados 50 dos 578 setores da base censitária do Instituto Brasileiro de Geografia e Estatística (IBGE) em São Luís, a partir de listagem ordenada dos mesmos, com probabilidade proporcional ao número de domicílios particulares ocupados, tendo como base o censo de 1991. No segundo estágio foram sorteados os quarteirões e, no terceiro estágio, foram sorteados os domicílios para serem visitados após a realização de censo no quarteirão sorteado, ambos por amostragem aleatória simples. Em cada setor foram visitados 40 domicílios, com média de 15 crianças por setor. O percentual de recusas e ausências foi de $7 \%$, após três repasses em dias e horários diferentes. Não se fez substituição de domicílios nos casos de perda. Foi utilizado questionário padronizado para coleta de dados, respondido pela mãe ou responsável, bastante similar ao utilizado em pesquisa nos estados do Nordeste (15).

As variáveis estudadas foram realização de puericultura, número de consultas de puericultura realizadas, época de início, categoria de atendimento, satisfação com a puericultura, gênero e idade da criança, escolaridade paterna e materna (em anos completos 
de freqüência à escola), renda familiar em salários mínimos, ocupação do chefe de família (não manual, manual especializada ou semi-especializada, manual não qualificada ou desempregado). A categorização da ocupação foi adaptada com base na Classificação Internacional Padrão de Ocupações (ISCO-68) (16). Foram estudadas ainda as variáveis idade materna, número de irmãos residindo no domicílio, número de moradores residindo na casa, posse de seguro-saúde (incluindo-se qualquer modalidade de assistência médica supletiva do tipo pré-pagamento) e morbidade referida nos 3 últimos meses.

Em relação à puericultura, calculouse a cobertura levando em consideração pelo menos uma visita ao médico no primeiro ano de vida, com finalidade de controle de saúde, para as crianças entre 1 e 23 meses de idade. Excluíram-se as crianças menores de 1 mês, pois estas poderiam ainda realizar consulta antes de completar 1 mês de vida. Para o cálculo da época de início da puericultura e do número consultas realizadas no primeiro ano de vida, foram utilizados os dados de crianças de 12 a 23 meses, para não se subestimar o número de consultas realizadas no primeiro ano e poder incluir como tendo feito puericultura as crianças que a iniciaram tardiamente. Calculou-se, também, a cobertura de puericultura para crianças de 12 a 23 meses, para fins de comparação com outros trabalhos.

A coleta de dados foi realizada de 15 de agosto a 25 de setembro de 1994 por 12 bolsistas do Conselho Nacional de Desenvolvimento Científico e Tecnológico (CNPq), estudantes universitários ou recém-graduados em Medicina ou Enfermagem. Os entrevistadores receberam treinamento de aplicação das entrevistas e participaram de estudo piloto. O trabalho de campo teve um supervisor (docente de ensino superior da área da saúde) para cada dois alunos.

A análise estatística incluiu cálculo da cobertura de puericultura e da razão de prevalências (risco relativo, RR). $\mathrm{O}$ risco foi sempre calculado em relação à categoria basal (categoria com menor risco para a não realização de consultas). As estimativas pontuais e por intervalo de confiança de $95 \%$ das proporções foram calculadas utilizando-se a opção CSAMPLE do programa Epi Info versão 6.02 (17), sendo o setor censitário a unidade primária de amostragem. O número de crianças estudadas por setor variou de duas a 33 , sendo a média igual a 15 . O efeito de desenho foi calculado para cada estimativa. Quando se utiliza amostragem por conglomerados, a variância das estimativas tende a ser maior em comparação à amostragem aleatória simples. Os intervalos de confiança são mais largos e a precisão das estimativas é, portanto, menor (17). Os cálculos do intervalo de confiança e do efeito de desenho levaram em conta estas diferenças no tamanho das unidades primárias de amostragem. Os cálculos assumiram amostragem com reposição, pois a fração amostral foi baixa (1\%), sendo estudadas 748 das 82403 crianças menores de 5 anos de São Luís (18). Foram consideradas para este trabalho 301 crianças de 1 a 23 meses.

No ajuste para fatores de confusão, empregou-se a regressão de Cox modificada por Breslow, obtendo-se estimativa da razão de prevalências ajustada e respectivo intervalo de confiança de $95 \%$. Breslow modificou a regressão de Cox impondo uma condição de duração constante de seguimento $(t=1)$ para todos os indivíduos, tornando este método, típico dos estudos de coorte, aplicável a estudos transversais $(19,20)$.

Foram utilizados dois processos de modelagem: o modelo completo e o modelo reduzido (21). A razão de prevalências obtida a partir do modelo completo foi uma estimativa ajustada, levando em conta todas as outras variáveis analisadas. $\mathrm{O}$ modelo reduzido foi obtido por meio do módulo em passos (stepwise), utilizando-se o processo de seleção para frente (forward). Todas as variáveis associadas com a não realização de consultas de puericultura em um nível de significância de 0,20 na análise não ajustada foram incluídas no modelo e ficaram apenas aquelas que permaneceram associadas a um nível de significância de pelo menos 0,10. A significância de cada variável no modelo foi verificada pelo teste da razão de verossimilhanças. Todas as variáveis foram incluídas na análise como categóricas.

\section{RESULTADOS}

A cobertura de puericultura foi de $80 \%$, considerando-se as informações referentes às crianças de 1 a 23 meses, e de $75 \%$, incluindo-se apenas as crianças de 12 a 23 meses. Dentre as 301 crianças de 1 a 23 meses de idade, 11 (4\%) foram excluídas porque suas mães não souberam informar sobre a realização de consultas de puericultura. Todas as crianças excluídas tinham entre 12 e 23 meses. Dessa forma, a amostra final incluiu 290 crianças. Das 147 mães de crianças de 12 a 23 meses, 146 informaram sobre a realização de consultas (tabela 1).

Dentre as mães ou responsáveis pelas crianças de 12 a 23 meses que freqüentaram consultas de puericultura, $110(75 \%)$ de 146 responderam sobre o número de consultas de puericultura realizadas. Destas crianças, $44 \%$ realizaram até seis consultas (tabela 1). A média de consultas de puericultura, dentre as crianças que as realizaram no primeiro ano de vida, foi de 7,8 .

No cálculo da época do início de puericultura foram utilizadas as informações das 110 crianças entre 12 e 23 meses. Iniciaram a puericultura na época adequada, isto é, antes de completar 1 mês de idade, 50\% destas crianças (tabela 1).

Oitenta e um por cento das crianças buscaram o Sistema Único de Saúde (SUS) para o atendimento de puericultura, sendo de $15 \%$ a utilização do seguro-saúde neste tipo de atendimento. A maioria, 233 mães de crianças entre 1 e 23 meses, respondeu sobre a satisfação com o atendimento de puericultura. O grau de satisfação foi razoável, pois $93 \%$ das mães se disseram satisfeitas, enquanto $3 \%$ se disseram mais ou menos satisfeitas e $4 \%$ se declararam insatisfeitas com o serviço (tabela 1).

No estudo dos fatores associados à não realização de puericultura, na análise não ajustada, não foram observadas diferenças segundo o gênero e a idade da criança. A freqüência à pueri- 
TABELA 1. Cobertura, número de consultas, mês de início, categoria de atendimento e satisfação com a puericultura em crianças, São Luís (MA), Brasil, 1994

\begin{tabular}{|c|c|c|c|c|}
\hline Indicador & $n$ & $\%$ & IC95\% & $\begin{array}{l}\text { Efeito de } \\
\text { desenho }\end{array}$ \\
\hline Puericulturaa $^{a}$ & & & & 1,812 \\
\hline Sim & 231 & 80 & $73,4-85,9$ & \\
\hline Não & 59 & 20 & $14,1-26,6$ & \\
\hline Total & 290 & 100 & & \\
\hline Número de consultas ${ }^{b}$ & & & & 1,599 \\
\hline Até 3 & 14 & 13 & $4,9-20,6$ & \\
\hline 4 a 6 & 34 & 31 & $20,6-41,2$ & \\
\hline 7 a 9 & 8 & 7 & $3,4-11,2$ & \\
\hline 10 a 12 & 13 & 12 & $3,5-18,6$ & \\
\hline Mais de 12 & 41 & 37 & $25,4-49,2$ & \\
\hline Total & 110 & 100 & & \\
\hline Idade ao início da puericultura & & & & \\
\hline$(\text { meses })^{b}$ & & & & 1,005 \\
\hline Menos de 1 & 55 & 50 & $40,6-59,4$ & \\
\hline 1 a 3 & 50 & 45 & $36,7-54,2$ & \\
\hline 4 a 6 & 2 & 2 & $0,0-4,2$ & \\
\hline 6 a 11 & 2 & 2 & $0,0-4,3$ & \\
\hline 12 e mais & 1 & 1 & $0,0-2,7$ & \\
\hline Total & 110 & 100 & & \\
\hline Categoria de atendimento ${ }^{a}$ & & & & 1,629 \\
\hline Setor público do SUS ${ }^{c}$ & 174 & 74,4 & $67,2-81,5$ & \\
\hline Setor contratado do SUS ${ }^{c}$ & 15 & 6,4 & $3,4-9,4$ & \\
\hline Seguro-saúde & 35 & 15,0 & $8,6-21,3$ & \\
\hline Particular & 8 & 3,4 & $1,1-5,8$ & \\
\hline Outro & 2 & 0,9 & $0,0-2,0$ & \\
\hline Total & 234 & 100,0 & & \\
\hline Satisfação com o atendimento & & & & \\
\hline de puericultura ${ }^{a}$ & & & & 0,927 \\
\hline Sim & 216 & 93 & $89,5-95,9$ & \\
\hline Mais ou menos & 7 & 3 & $0,7-5,4$ & \\
\hline Não & 10 & 4 & $1,9-5,4$ & \\
\hline Total & 233 & 100 & & \\
\hline
\end{tabular}

a Indicadores calculados em relação às crianças de 1 a 23 meses.

${ }^{b}$ Indicadores calculados em relação às crianças de 12 a 23 meses.

c SUS = Sistema Único de Saúde.

cultura foi maior para crianças cujos pais tinham freqüentado 9 ou mais anos de escola (90\%) e menor para aquelas cujos pais tinham até 4 anos de escolaridade $(65 \%)$. O risco de uma criança de pai com até 4 anos de escolaridade não freqüentar a puericultura foi 3,4 vezes maior em relação às demais. Crianças de mães que freqüentaram até 4 anos de escola apresentaram menor freqüência às consultas.

As crianças com renda familiar de mais de três salários mínimos tiveram maior cobertura $(90 \%)$ do que as com renda de até um salário mínimo (73\%). $\mathrm{O}$ risco de não freqüentar puericultura foi 2,7 vezes maior para as crianças com renda familiar de um até três salá- rios mínimos e 2,8 vezes maior para as crianças com renda familiar de menos de um salário mínimo, em relação às mais ricas. As crianças de famílias com chefes trabalhando em ocupações não qualificadas ou desempregados freqüentaram menos a puericultura (74\%), quando comparadas às crianças de famílias cujos chefes trabalhavam em ocupações não manuais $(90 \%)$ (tabela 2). A realização de consultas não mostrou diferenças em relação à idade materna, número de moradores no domicílio e morbidade. As crianças com até dois irmãos compareceram mais à puericultura $(82 \%)$ do que as demais (50\%). O risco de uma criança com mais de dois irmãos no domicílio não freqüentar puericultura foi de 2,8 em relação às outras. As crianças com seguro-saúde freqüentaram mais as consultas $(90 \%)$ do que as que não o possuíam (77\%) (tabela 3).

Após o ajuste para fatores de confusão, no modelo completo, somente a baixa escolaridade do pai (até 4 anos de estudo) explicou a não freqüência à puericultura (tabelas 2 e 3). No modelo reduzido, maior número de irmãos no domicílio e baixa escolaridade paterna estiveram associados com a não realização de consultas de puericultura (tabela 4).

\section{DISCUSSÃO}

A cobertura de puericultura em crianças de 1 a 23 meses $(80 \%)$ e de 12 a 23 meses (75\%) em São Luís foi bastante inferior à cobertura de $98 \%$ relatada para as cidades brasileiras de São Paulo, em 1988, e de Londrina, em 1990. Dentre as crianças que freqüentaram os serviços, a média de consultas, situada em 7,8, foi comparável à de São Paulo $(7,7)$ e inferior à de Londrina $(10,8)$, porém ficou abaixo da meta de nove consultas para o primeiro ano de vida. O início da puericultura em São Luís foi mais tardio (80\% até o primeiro mês de vida) em comparação com Londrina, onde 99\% iniciaram a puericultura no primeiro mês $(10,12)$.

Da mesma forma como constatado na pesquisa nacional por amostra de domicílios (PNAD) de 1981 (22), a maior parte dos atendimentos preventivos foi realizada em estabelecimentos públicos (74\% no caso das consultas de puericultura). Apesar de $21 \%$ das crianças possuírem seguro-saúde, apenas $15 \%$ fizeram uso do seguro nas consultas de puericultura, talvez devido à necessidade de cumprimento de carências contratuais.

Como no Chile, em 1983 (6), no presente estudo a taxa de insatisfação com os serviços de puericultura foi baixa. $\mathrm{Ou}$ as mães realmente gostaram da puericultura, ou este índice se deveu à pequena exigência que as mães fazem dos serviços de saúde; ou, ainda, se deveu à dificuldade desta pergunta di- 
TABELA 2. Utilização de consultas de puericultura por crianças de 1 a 23 meses segundo gênero, idade da criança e alguns indicadores socioeconômicos, São Luís (MA), Brasil, 1994

\begin{tabular}{|c|c|c|c|c|c|c|}
\hline \multirow[b]{3}{*}{ Variáveis } & \multicolumn{4}{|c|}{ Utiliza puericultura } & \multirow{3}{*}{$\begin{array}{c}\text { Razão de } \\
\text { prevalências } \\
\text { bruta } \\
\text { (IC95\%) }\end{array}$} & \multirow{3}{*}{$\begin{array}{l}\text { Razão de } \\
\text { prevalências } \\
\text { ajustada } \\
(\mathrm{IC} 95 \%)^{\mathrm{a}}\end{array}$} \\
\hline & \multicolumn{2}{|c|}{ Não } & \multicolumn{2}{|c|}{ Sim } & & \\
\hline & $n$ & $\%$ & $n$ & $\%$ & & \\
\hline \multicolumn{7}{|l|}{ Gênero } \\
\hline Masculino & 27 & 19 & 112 & 81 & & \\
\hline Feminino & 32 & 21 & 119 & 79 & $1,09(0,69-1,72)$ & $1,06(0,60-1,87)$ \\
\hline \multicolumn{7}{|l|}{ Idade da criança } \\
\hline 1 a 11 meses & 23 & 16 & 121 & 84 & & \\
\hline 12 a 23 meses & 36 & 25 & 110 & 75 & $1,54(0,97-2,47)$ & $1,47(0,80-2,69)$ \\
\hline \multicolumn{7}{|l|}{ Escolaridade paterna } \\
\hline 9 ou mais anos de estudo & 13 & 10 & 113 & 90 & & \\
\hline 5 a 8 anos de estudo & 19 & 21 & 70 & 79 & $2,07(1,08-3,97)$ & $1,50(0,68-3,27)$ \\
\hline Até 4 anos de estudo & 19 & 34 & 36 & 65 & $3,35(1,78-6,29)$ & $2,36(1,02-5,43)$ \\
\hline \multicolumn{7}{|l|}{ Escolaridade materna } \\
\hline 9 ou mais anos de estudo & 18 & 14 & 108 & 86 & & \\
\hline 5 a 8 anos de estudo & 26 & 24 & 81 & 76 & $1,70(1,04-3,51)$ & $1,04(0,51-2,13)$ \\
\hline Até 4 anos de estudo & 15 & 27 & 40 & 73 & $1,91(1,04-3,51)$ & $0,95(0,41-2,19)$ \\
\hline \multicolumn{7}{|l|}{ Renda familiarb } \\
\hline > 3 salários mínimos & 9 & 10 & 84 & 90 & & \\
\hline 1 a 3 salários mínimos & 33 & 26 & 92 & 74 & $2,73(1,37-5,42)$ & $1,86(0,78-4,47)$ \\
\hline Até 1 salário mínimo & 17 & 27 & 46 & 73 & $2,79(1,33-5,86)$ & $1,39(0,48-4,08)$ \\
\hline \multicolumn{7}{|l|}{ Ocupação } \\
\hline Não manual & 6 & 10 & 55 & 90 & & \\
\hline $\begin{array}{l}\text { Manual especializada ou } \\
\text { semi-especializada }\end{array}$ & 25 & 20 & 97 & 79 & $2,08(0,90-4,81)$ & $1,35(0,52-3,50)$ \\
\hline $\begin{array}{l}\text { Manual não qualificada ou } \\
\text { desempregado }\end{array}$ & 28 & 26 & 78 & 74 & $2,69(1,18-6,12)$ & $1,27(0,46-3,47)$ \\
\hline
\end{tabular}

a Razão de prevalências ajustada segundo modelo completo de regressão.

${ }^{\text {b }} \mathrm{Na}$ época do estudo, o salário mínimo correspondia a 70 dólares.

TABELA 3. Utilização de consultas de puericultura por crianças de 1 a 23 meses segundo alguns indicadores demográficos, posse de seguro-saúde e morbidade, São Luís (MA), Brasil, 1994

\begin{tabular}{|c|c|c|c|c|c|c|}
\hline \multirow[b]{3}{*}{ Variáveis } & \multicolumn{4}{|c|}{ Utiliza puericultura } & \multirow{3}{*}{$\begin{array}{c}\text { Razão de } \\
\text { prevalências } \\
\text { bruta } \\
(\mathrm{IC} 95 \%)\end{array}$} & \multirow{3}{*}{$\begin{array}{l}\text { Razão de } \\
\text { prevalências } \\
\text { ajustada } \\
(\text { IC95\%) }\end{array}$} \\
\hline & \multicolumn{2}{|c|}{ Não } & \multicolumn{2}{|c|}{ Sim } & & \\
\hline & $n$ & $\%$ & $n$ & $\%$ & & \\
\hline \multicolumn{7}{|l|}{ Idade materna } \\
\hline$<20$ anos & 9 & 18 & 40 & 82 & & \\
\hline 20 anos e mais & 49 & 20 & 190 & 79 & $1,12(0,59-2,12)$ & $1,05(0,45-2,42)$ \\
\hline \multicolumn{7}{|l|}{ Número de irmãos } \\
\hline 0 a 2 & 48 & 18 & 220 & 82 & & \\
\hline 3 a 9 & 11 & 50 & 11 & 50 & $2,79(1,71-4,56)$ & $2,10(0,89-4,94)$ \\
\hline \multicolumn{7}{|l|}{ Moradores na casa } \\
\hline 1 a 5 & 29 & 18 & 136 & 82 & & \\
\hline 6 a 18 & 30 & 24 & 95 & 76 & $1,37(0,87-2,15)$ & $1,15(0,61-2,19)$ \\
\hline \multicolumn{7}{|l|}{ Seguro-saúde } \\
\hline Sim & 6 & 10 & 56 & 90 & & \\
\hline Não & 53 & 23 & 175 & 77 & $2,40(1,08-5,33)$ & $1,23(0,46-3,34)$ \\
\hline \multicolumn{7}{|c|}{$\begin{array}{l}\text { Morbidade referida nos últimos } \\
3 \text { meses }\end{array}$} \\
\hline Não & 20 & 18 & 89 & 82 & & \\
\hline Sim & 39 & 21 & 142 & 78 & $1,17(0,72-1,91)$ & $0,95(0,51-1,76)$ \\
\hline
\end{tabular}

${ }^{a}$ Razão de prevalências ajustada segundo modelo completo de regressão. reta em captar a verdadeira insatisfação. Ademais, as mães foram entrevistadas por estudantes e profissionais de saúde e podem não ter ficado à vontade para revelar a sua insatisfação (23). Quando se estabelece um clima de confiança na entrevista, os pacientes falam mais de como vêem os serviços. Alguns autores referem que os pacientes costumam se queixar das consultas rápidas, do tempo de espera para o atendimento, das horas inadequadas de atendimento que coincidem com o horário de trabalho, da má comunicação que se estabelece com médicos e profissionais de saúde e dizem que gostariam de receber maiores explicações sobre a doença (24).

O fato de as crianças com maior número de irmãos terem realizado a puericultura em menor proporção do que as crianças de famílias menos numerosas provavelmente ilustra a dificuldade que as tarefas domésticas relativas ao cuidado de vários filhos colocam para a mãe no sentido de levar as crianças à puericultura. É possível que a vontade de levar as crianças à puericultura esteja sendo constrangida pela carga de trabalho a que muitas mulheres estão expostas no domicílio, sem muitas vezes poderem contar com ajuda do pai da criança, de familiares e de vizinhos. $\mathrm{O}$ acesso a outras facilidades, como creches, também é difícil para determinados segmentos da população. Isto indica a necessidade de mais estudos no grupo de crianças não usuárias de serviços, centrados na identificação dos motivos do não comparecimento à puericultura.

Em estudos semelhantes, realizados no Chile e no Panamá, também foi identificada esta associação entre maior número de filhos e menor freqüência à puericultura $(6,7)$. A maior experiência das mães com mais filhos pode ser um dos fatores responsáveis por este fato. É possível que estas mães achem que já sabem resolver os problemas de seus filhos e que, dessa forma, optem por deixar de procurar o médico, por exemplo por razões econômicas. A persistência da associação entre maior número de filhos e menor número de consultas realizadas, após o controle dos fatores de confusão, fala 
TABELA 4. Modelo reduzido da regressão de Cox para consultas de puericultura em crianças de 1 a 23 meses, São Luís (MA), Brasil, 1994

\begin{tabular}{lc}
\hline \multicolumn{1}{c}{ Variáveis } & Razão de prevalências (IC95\%) \\
\hline Idade de 12 a 23 meses & $1,44(0,81-2,55)$ \\
3 a 9 irmãos & $2,32(1,14-4,73)^{\mathrm{a}}$ \\
Renda familiar de mais de 1 até 3 salários mínimos & $1,93(0,84-4,41)$ \\
Renda familiar até 1 salário mínimo & $1,52(0,59-3,95)$ \\
Pai com 5 a 8 anos de estudo & $1,64(0,79-3,42)$ \\
Pai com até 4 anos de estudo & $2,40(1,10-5,24)^{\mathrm{a}}$ \\
\hline
\end{tabular}

a Variáveis significativas em nível de 0,05.

${ }^{\text {b }} \mathrm{Na}$ época do estudo, o salário mínimo correspondia a 70 dólares.

a favor de uma ação independente deste fator.

Não foi encontrada associação entre idade da mãe e consultas de puericultura, apesar de outros trabalhos terem identificado esta associação $(6,7)$.

A escolaridade paterna esteve associada com a realização de consultas preventivas, ao contrário da escolaridade materna. A escolaridade paterna está altamente relacionada à escolaridade materna e ambas são indicadores da questão educacional no núcleo familiar. Isto confirma a importância dos fatores educacionais na aquisição de comportamentos preventivos, como a realização de puericultura. A associação entre menor escolaridade do pai, da mãe ou do chefe da família e menor freqüência à puericultura foi descrita no Panamá (7), em Burkina Fasso (8) e no Brasil, em São Paulo e em Londrina $(10,12)$. Tal como encontrada neste trabalho, a associação entre posse de seguro-saúde e maior freqüência aos controles de puericultura também foi descrita nos Estados Unidos (9).

A associação entre renda familiar e maior freqüência à puericultura também foi encontrada no Panamá (7) e em certas cidades no Brasil, como Pelotas (RS) (11) e Londrina (PR) (12). Estudos em outros países não encontraram associação entre maior renda e maior uso de consultas, como no Chile (6) e em Burkina Fasso (8), o que fala a favor de um sistema de saúde mais eqüitativo nestes últimos países.

No presente estudo, o desaparecimento do efeito da renda familiar, do tipo de ocupação do chefe de família e do seguro-saúde em relação à puericultura, após o ajuste para fatores de confusão, aponta para a possibilidade de que barreiras de acesso econômico à puericultura hoje sejam de menor importância em São Luís. A expansão da rede pública básica de serviços de saúde no município foi grande nos últimos 10 anos. É provável que, atualmente, a oferta de serviços de puericultura não esteja sendo um fator de grande constrangimento à realização de consultas. A persistência da associação entre não realização de puericultura e escolaridade, após o controle dos fatores de confusão, sugere que razões educativas e culturais são os fatores que melhor explicam a não realização da puericultura nesta população. Isto coloca a necessidade de serem conduzidos novos estudos no sentido de identificarem-se os fatores mediadores entre a baixa escolaridade e a não realização da puericultura, ou seja, os fatores envolvidos na decisão de procurar ou não os serviços (24). Talvez, em muitos casos, as redes sociais (família, parentesco e amizade) sejam fatores mais importantes para o uso de serviços de saúde do que a classe social (25).

$\mathrm{O}$ ajuste para fatores de confusão foi realizado com o indicador cobertura. É provável que as desigualdades sociais, no que diz respeito à qualidade dos serviços (medida pelo início precoce e número de consultas), sejam maiores do que demonstra o presente estudo com base apenas na realização de pelo menos uma consulta preventiva.

Os resultados do presente trabalho indicam, ainda, que são necessários esforços para que todas as crianças possam iniciar as consultas preventivas com 1 mês de idade. A desigualdade na realização de consultas de puericultura em relação à escolaridade paterna e ao número de filhos indica a existência de barreiras e constrangimentos que estão atuando mais fortemente sobre os grupos de menor escolaridade e sobre famílias mais numerosas. $\mathrm{O}$ reforço às atividades educativas $\mathrm{e}$, talvez, aos programas de planejamento familiar, é uma estratégia importante no sentido de aumentar-se a cobertura de puericultura no município e reduzir-se a desigualdade aqui apontada.

Agradecimentos. Este estudo foi financiado pela Fundação de Amparo à Pesquisa do Estado do Maranhão (Processo no. 446/93), Conselho Nacional de Desenvolvimento Científico e Tecnológico $(\mathrm{CNPq})$, Coordenação de Aperfeiçoamento de Pessoal de Nível Superior (CAPES), Secretaria de Estado da Saúde e Secretaria Municipal de Saúde de São Luís. Agradecemos as valiosas sugestões dos professores Marco Antônio Barbieri, Antônio de Azevedo Barros Filhos e Afonso Diniz Costa Passos. Agradecemos ainda a Zilmar Alves Ferreira do IBGE, Maranhão, pelo fornecimento dos mapas dos setores censitários. 


\section{REFERÊNCIAS}

1. Ministério da Saúde. Atendimento integral à saúde e desenvolvimento da criança: cartão da criança. Brasília: Ministério da Saúde; 1992.

2. Nordberg E. Household health surveys in developing countries: could more use be made of them in planning? Health Policy Plan 1988;3(1):32-39.

3. Casanova C, Starfield B. Hospitalizations of children and access to primary health care: a cross-national comparison. Int J Health Serv 1995;25(2):283-294.

4. Cohen MM, MacWilliam L. Measuring the health of the population. Med Care 1995;33 (Suppl 12):DS21-DS42.

5. Kaempffer AMR, Medina E. Morbilidad y atención médica infantil en el Gran Santiago. Rev Chil Pediatr 1980; 51(5):355-369.

6. Medina EL, Kaempffer AMR, Cumsille FG, Medina RK. Factores de importancia en la demanda de atención médica infantil. Rev Chil Pediatr 1985;56(2):113-119.

7. Huezo CM, Monteith RS, Naar H, Morris L. Utilización de los servicios de salud de la madre y el niño y cobertura de la inmunización en Panamá. Bol Oficina Sanit Panam 1982; 93(3):191-206.

8. Nougtara A, Sauerborn R, Oepen C, Diesfeld $\mathrm{HJ}$. Assessment of $\mathrm{MCH}$ services offered by professional and community health workers in the district of Solenzo, Burkina Faso. I. Utilization of MCH services. J Trop Pediatr 1989; 35(Suppl 1):2-9

9. Holl JL, Szilagyi PG, Rodewald LE, Byrd RS, Weitzman ML. Profile of uninsured children in the United States. Arch Pediatr Adolesc Med 1995;149(4):398-406.
10. Monteiro CA, Medina MCG, Benicio MHD'A, Meyer M. Estudo das condições de saúde das crianças do Município de São Paulo (Brasil), 1984/1985: XI—Cobertura e qualidade da assistência materno-infantil. Rev Saude Publica 1988;22(3):170-178.

11. Costa JSD, Victora CG, Barros FC, Halpern B, Horta BL, Manzolli P. Assistência médica materno-infantil em duas coortes de base populacional no Sul do Brasil: tendências e diferenciais. Cad Saude Publica 1996;12(Suppl 1): 59-66.

12. Campos JJB. Estudo das condições de saúde das crianças do município de Londrina [dissertação]. São Paulo (SP): Faculdade de Medicina de Ribeirão Preto da Universidade de São Paulo; 1992.

13. Fundação Instituto Brasileiro de Geografia e Estatística. Contagem da População 1996: dados preliminares. Brasil, Rio de Janeiro, Brasil: IBGE; 1997.

14. Kalton G. Introduction to survey sampling. Beverly Hills, California: Sage; 1983.

15. Barros FC, Victora CG. Epidemiologia da saúde infantil. São Paulo: Hucitec/Unicef; 1991.

16. Olsen J, Frische G. Social differences in reproductive health. Scand J Soc Med 1993;21(2): 90-97.

17. Dean AG, Dean JA, Coulombier D, Brendel KA, Smith DC, Burton AH, et al. Epi Info, Version 6: a word processing, database, and statistics program for epidemiology on microcomputers. Atlanta, Georgia: Centers for Disease Control and Prevention; 1994.

18. Instituto Brasileiro de Geografia e Estatística. Censo Demográfico de 1991. Situação demo- gráfica, social e econômica: primeiras considerações: Estado do Maranhão. Rio de Janeiro, Brasil: IBGE; 1995.

19. Breslow NE. Covariance analysis of censored survival data. Biometrics 1974;30(1):89-99.

20. Cox DR. Regression models and life tables. J R Stat Soc B 1972;34(2):187-220.

21. Kleinbaum DG. Survival analysis: a self-learning text. New York: Springer-Verlag; 1996.

22. Simões CCS, Oliveira LAP, Pereira NOM. A oferta e a utilização de serviços de saúde. Em: Silva RMR, org. Perfil estatístico de crianças e mães no Brasil: situação de saúde, 1981. Rio de Janeiro: IBGE; 1984: pp. 65-86.

23. Kroeger A. Health interview surveys in developing countries: a review of methods and results. Int J Epidemiol 1983;12(4):465-481.

24. Sauerborn R, Nougtara A, Sorgho G, Bidiga J, Tiebelesse L, Diesfeld HJ. Assessment of $\mathrm{MCH}$ services in the district of Solenzo, Burkina Faso: II-Acceptability. J Trop Pediatr 1989;35(Suppl 1):10-13.

25. Osman LM, Dunt D. Factors influencing mothers' decision to consult a general practitioner about their children's illnesses. Br J Gen Pract 1995;45(395):310-312.

Manuscrito recebido em 23 de julho de 1998. Aceito em versão revisada em 16 de março de 1999.

ABSTRACT Pediatric care is fundamental to preventing several diseases during the early years of childhood. Early medical care and having at least nine checkups in the first year of life are the ideal in pediatric care. It is essential to periodically assess whether or not these goals are being met. In 1994, a cross-sectional study was carried out using three-stage cluster sampling to measure coverage of pediatric care and factors associated with that care among children in the city of São Luís, in the state of Maranhão, Brazil. For data collection, a standardized questionnaire was used with the mother of the child or another person responsible for the child. The percentage of those refusing to participate or who were absent was $7 \%$. This work analyzes data concerning 290 children between 1 and 23 months of age. The coverage estimates and 95\% confidence intervals took into consideration possible effects of the study design. Cox's regression, modified for cross-sectional studies, was used to estimate adjusted relative risks. Pediatric care coverage for the population studied was $80 \%$. The children had an average of 7.8 checkups in the first year; $44 \%$ of the children had six or fewer checkups during the first year of life, well below the recommended number. Eighty-one percent of the children were seen in the Brazilian public health care system (Sistema Unico de Saude, SUS), and only 15\% used health insurance. After adjusting for confounding factors, the factors "greater number of siblings at home" and "father with four or fewer years of schooling" were associated with not having checkups. After adjustment, family income was not associated with the number of checkups. Encouraging educational activities and perhaps also family planning programs are important strategies for increasing the coverage of pediatric care in the city of São Luís. 